\title{
Inflammation in Cardiovascular Tissue Engineering: The Challenge to a Promise: A Minireview
}

\author{
Agneta Simionescu, Jason B. Schulte, George Fercana, and Dan T. Simionescu \\ Biocompatibility and Tissue Regeneration Laboratory, Department of Bioengineering, Clemson University, 304 Rhodes Center, \\ Clemson, SC 29634, USA \\ Correspondence should be addressed to Dan T. Simionescu, dsimion@clemson.edu
}

Received 24 March 2011; Accepted 10 May 2011

Academic Editor: Adrian Chester

Copyright ( $\odot 2011$ Agneta Simionescu et al. This is an open access article distributed under the Creative Commons Attribution License, which permits unrestricted use, distribution, and reproduction in any medium, provided the original work is properly cited.

Tissue engineering employs scaffolds, cells, and stimuli brought together in such a way as to mimic the functional architecture of the target tissue or organ. Exhilarating advances in tissue engineering and regenerative medicine allow us to envision in vitro creation or in vivo regeneration of cardiovascular tissues. Such accomplishments have the potential to revolutionize medicine and greatly improve our standard of life. However, enthusiasm has been hampered in recent years because of abnormal reactions at the implant-host interface, including cell proliferation, fibrosis, calcification and degeneration, as compared to the highly desired healing and remodeling. Animal and clinical studies have highlighted uncontrolled chronic inflammation as the main cause of these processes. In this minireview, we present three case studies highlighting the importance of inflammation in tissue engineering heart valves, vascular grafts, and myocardium and propose to focus on the endothelial barrier, the "final frontier" endowed with the natural potential and ability to regulate inflammatory signals.

\section{Introduction}

Biomedical engineers in the cardiovascular tissue engineering (CVTE) arena dare to "boldly go where no man has gone before"; they mix scaffolds with cells, add mechanical stimuli, growth factors, and other ingredients, culture the constructs in vitro for maturation and ... voilá: a newly created surrogate structure ready to replace an inflamed, thrombotic, atherosclerotic, calcified, or infected cardiovascular tissue [1]. Tissue engineering and regenerative medicine are at the footsteps of clinical translation and hold great therapeutic potential. However, progress in the field is critically hampered by uncontrolled, chronic implant-host interactions and more specifically by chronic inflammation.

When challenged by an implanted biomaterial, the body selects one or more of its three defense mechanisms existent in the "armamentarium": hemostasis/coagulation, immune reactions, and inflammation [2]. While we have the ability to control the first two mechanisms relatively well using drugs, chronic cardiovascular inflammation is more difficult to manage. Moreover, the clinical consequences of chronic inflammation including uncontrolled cell proliferation, fibrosis, calcification, and sclerosis are almost impossible to treat pharmaceutically $[3,4]$.

As we will describe in this minireview, technical challenges in CVTE are plentiful, but the "final frontier" is the healthy, quiescent endothelium [5]. This monolayer of cells that naturally covers all blood-contacting tissues acts as a dynamic and selective barrier by maintaining a nonthrombogenic surface, controls the transfer of molecules across the vascular wall, modulates blood flow and vascular resistance, regulates immune and inflammatory reactions and also interacts with underlying cells to regulate their growth and proliferation. The activation of the endothelium by cytokines, bacterial products, hemodynamic forces, lipids, and other agents induces expression of a new and radically different cell phenotype. Activated endothelium expresses new adhesion molecules on its surface and secretes chemokines, growth factors, vasoactive mediators, and coagulation proteins. Dysfunctional endothelium becomes 
adhesive to inflammatory cells, exposes thrombogenic surfaces, and thus promotes inflammation, atherosclerosis, and thromboembolism [6-8] Activation of other cardiovascular cells such as vascular smooth muscle cells and cardiac fibroblasts also contributes significantly to cardiovascular pathology by initiating intimal hyperplasia [9] and cardiac fibrosis [10], respectively.

Overall, the presence, integrity, and state of activation of an endothelial surface at the implant-host interface can "make or break" a tissue-engineered cardiovascular device. It is, thus, clear that the secret to successful CVTE is gaining control over inflammation by modulating the endothelium, the "ultimate interface".

\section{Inflammation in Cardiovascular Tissue Engineering}

After implantation, cardiovascular devices typically undergo a process similar to wound healing [3]. Following an initial blood-material interaction where fibrin is deposited on the luminal surface, inflammatory processes occur around the implanted construct. In initial stages, neutrophils and monocytes migrate to the interface between the implant surface and the injured tissue. During the granulation phase, phagocytes remove debris due to trauma and then provide signals for fibroblasts and smooth muscle cells to start remodeling. This phase lasts 2-3 weeks in humans and ideally will culminate with complete healing. However, the inflammatory response may continue for months or years and, thus, may lead to chronic inflammation. The consequences of this deleterious process include intimal thickening, tissue overgrowth (pannus formation), foreign body reactions, granulation, fibrosis, and ectopic calcification. The mechanisms of these pathological phenomena are not fully understood, but it is known that monocytes/macrophages are observed within implanted constructs until they fully degrade or, if the implant is not degradable, for the life of the implant [4]. Mineralization of synthetic or biologic scaffolds is end-stage pathology, generally irreversible and untreatable. It is also commonly accepted that calcification may not occur if scaffolds degrade slowly and the matrix is capable of remodeling [11]. In most studies on chronic inflammation reported to date, a "diffuse mononuclear infiltrate" was described together with "frustrated macrophages" a.k.a., foreign body giant cells [12]. The latter secrete large amounts of proteases and reactive oxygen species which in turn promote implant degradation, loosening, and eventual failure. Recent evidence suggests that infiltrated monocytes/macrophages produce important cytokines, growth factors, and matrix-remodeling proteases (specifically metalloproteinases). Monocytes/macrophages express markers typical of proinflammatory macrophages (type M1) or remodeling macrophages (type M2) depending on whether the implanted scaffolds are degradable or not [13-16]. Additionally, recruited monocytes release vascular endothelial growth factors and thus may be involved in undesired neovascularization of CVTE implants [17].
Clearly, a better understanding of the underlying pathways appears crucial for controlling the fate of implants and modulating inflammatory reactions in such a way as to induce implant healing and remodeling as compared to fibrosis and/or degeneration. One way of approaching, this issue is developing imaging modalities and discovering new biomarkers of inflammation which would help further understanding of inflammatory diseases and discerning events related to inflammation in CVTE implants [18]. Examples include the detection of vascular cell adhesion molecules, markers for proteases, integrin labeling and uptake of oxidized low-density lipoprotein in atherosclerotic lesions [19-22]. Circulating C-reactive protein has become particularly favored as an inflammatory marker due to its long half-life and chemical stability [23-25] in addition to its ability to predict cardiovascular events [26] and evaluate the effectiveness of clinical anti-inflammatory treatment options $[7,27]$.

\section{Scaffolds for Cardiovascular Tissue Engineering}

Tissue engineering (TE), aided by emerging stem cell technology, holds immense potential for the treatment of cardiovascular (CV) diseases, as progress has been made in engineering the various components of the CV system, including blood vessels, heart valves, and cardiac muscle [28-31]. The goal of CVTE is to create or regenerate a functional structure populated with cells capable of continuously remodeling the extracellular matrix (ECM). Optimal replacements for failed CV components would be biocompatible tissues that have the potential to rapidly restore the lost function and slowly regenerate by remodeling. This is a bioengineer's dream come true and increasingly more reports show that we are slowly getting closer to viable solutions. The specific concepts of TE include the creation of a suitably shaped scaffold, repopulation with the appropriate cells (endothelium, smooth muscle cells, fibroblasts), ensuring that cells are placed in their appropriate tissue "niche" (e.g., lumen, media, adventitia), promotion of neovascularization (where needed), and dynamic mechanical conditioning [32] to slowly adapt cells to loads and prepare constructs for implantation. Scaffolds for CVTE must be noncytotoxic, biocompatible, biodegradable with safe by-products, and highly porous yet mechanically stable for the appropriate functions [33]. Bioscaffolds derived from xenogeneic ECM as well as synthetic polymers have been used in numerous TE applications. ECM is the natural scaffold for tissue and organ morphogenesis, maintenance, and reconstruction following injury, and is associated with constructive tissue remodeling. The $3 \mathrm{D}$ organization of its components and the complexity of the composition clearly distinguish the ECM from synthetic scaffolds [34]. The processing of ECM biomaterials for medical use involves decellularization of mammalian tissues in order to remove all epitopes associated with cells, especially the terminal galactose alpha 1,3 galactose (alpha-Gal) [35], expressed on the cell surface of all mammals except those of humans and 
old world primates [36]. It is known from earlier studies that pure collagen scaffolds degrade slowly and do not calcify in subdermal implantation models [37-39]. Aldehyde crosslinked ECM calcifies after implantation [40], thus, limiting the use of glutaraldehyde-treated valve bioprostheses. Notably, increasing the extent of matrix cross-linking by adding amine bridges [41] significantly reduced calcification when tested in a variety of animal models [42-45].

At early stages, scaffolds are infiltrated by macrophages which degrade ECM slowly by means of secreted matrix metalloproteinases (MMPs); at later stages scaffolds are infiltrated by fibroblasts which initiate repair and remodeling processes. Although degrading collagen scaffolds do not accumulate calcium deposits in vivo, degrading elastin scaffolds have a natural tendency to calcify unless stabilized [46]. While the mechanisms of this process are still under investigation, it is evident that matrix cytokines ("matrikines") such as elastin peptides can induce osteogenic responses in smooth muscle cells and fibroblasts and thus mediate calcification [47]. Thus it is apparent that there is a dire need for a method to stabilize elastin. Polyphenols such as tannic acid and its more stable core compound, pentagalloyl glucose (PGG) was shown to bind tightly to elastin and in doing so stabilizes elastin fibers sufficiently to reduce degeneration and calcification in animal models of aneurysms and in bioprosthetic heart valves [48-51]. In recent years we have extended the use of PGG to ECM scaffold stabilization for tissue engineering heart valves [37], and vascular grafts [38]. Notably, when tested in vivo, PGGstabilized acellular porcine pericardium and carotid arteries degraded at a slower rate than unstabilized scaffolds, did not calcify and underwent remodeling, suggesting that PGG could serve as an ideal stabilization agent for biological scaffolds [37, 38].

\section{Challenges in Cardiovascular Tissue Engineering}

Effective clinical application of the TE scenario described above raises a series of challenges linked to (1) the preexisting pathology, (2) the surgical procedure, and (3) the nature of the implant.

Little is known about the effect of the pathological status of a tissue, organ, or patient on the fate of CVTE devices and constructs. However it is reasonable to believe that the preexisting pathology or existing risk factors would influence long-term outcomes of device implantation. For example, implantation of a vascular graft in an atherosclerosis-prone patient results in decreased patency [52].

The surgical procedure may also affect host reactivity. In the first several weeks, surgery-related tissue trauma (much unrelated to the implant itself) induces an expected inflammatory response coupled to a wound healing reaction. The response follows the known pathways of inflammation followed by repair and healing. This portion of the implanthost interaction time line is necessary to heal the excised tissues and establish a "working interface" between the implant and host. Since the extent of the host response depends on the degree of surgical injury, more and more CVTE approaches envision devices that could be delivered and implanted using less traumatic, minimally invasive (percutaneous, laparoscopic, endovascular) approaches [53].

The nature of the implant could evidently have a great impact on its clinical outcome. Ideally, the CVTE implant should not be immunogenic, nor should it induce thromboembolic complications, or excessive and prolonged inflammation. Unfortunately, few biomaterials exist which can be considered completely inert. For example, ePTFE and Dacron vascular grafts function well in large diameter graft applications without endothelial cell coverage but when used in peripheral applications, one half of them occlude within the first five years of implantation [54].

A viable endothelium can drastically alter the outcomes of synthetic prostheses; this was demonstrated by in vitro endothelialization of ePTFE grafts, procedure pioneered by Prof. Peter Zilla in South Africa more than 20 years ago [55]. In this process, the patients' own endothelial cells were harvested and grown on synthetic ePTFE grafts prior to implantation. This resulted in an improvement in graft patency to values similar to those obtainable with autologous vessels [56].

Thus, to meet these challenges, novel CVTE approaches should take into account preexisting pathology by "personalizing" implants to each patient. This includes use of autologous cells and specific implants developed for specific disease states. We also need to develop implants that attempt to reduce surgical trauma to a minimum and prepare noninflammatory materials which are protected by a healthy endothelial layer. The following sections provide more details on inflammation and other challenges associated with use of TE heart valves, vascular grafts and myocardium.

\section{Heart Valve Tissue Engineering}

The hallmarks of valve pathology are fibrosis in rheumatic heart disease and calcification in aortic stenosis [11]. Postinflammatory scarring is one of the most cited mechanisms of valvular pathology; this denotes correlations with inflammation, but very little is known about the specific mechanisms involved in the onset and progression of heart valve pathology [6]. Jian et al. showed that as a response to injury, calcific degeneration in human valves is characterized by localized endothelial damage, macrophage infiltration, MMP secretion and activation, interstitial cell transformation into an osteogenic phenotype, apoptosis and calcification of devitalized cells and some processes possibly mediated by TGF-beta1 [47, 57]. Information obtained from human patient samples are just a snapshot in time and in most circumstances reflect the end stages of valvular disease. Currently, there is no effective medication available to limit, progression of heart valve diseases, and the most common treatment is their surgical replacement with mechanical devices or valves made from biological tissues. It is estimated at present that 275,000 valve replacements are performed annually worldwide using mechanical valves and tissue valves. However, the most physiological prostheses 
are the pulmonary autograft valves (transposition of the living patient's own pulmonary valve into the aortic position) and the allograft valves (sterilized, cryopreserved human cadaveric valves). Allografts exhibit excellent durability after implantation but are not readily available and represent only a small percentage of total valve replacements.

The main issue that emerged during clinical investigations of replacement heart valves was their limited durability which limits their use in young patients. Reoperation following valve replacement surgery, for the purpose of retrieving and replacing the defective device, is a relatively common event and occurs within 10-15 years after initial valve surgery [58]. Newer tissue valve models claim better performances [59], but this remains to be determined. Histological, ultra structural, and biochemical aspects of degenerated explanted tissue valves are similar to those of native human-diseased heart valves. The major processes that contribute to this "new pathology" of replacement heart valves are tissue calcification and mechanical damage. Calcification may occur independent of mechanical damage [60] but may also be accompanied by tissue abrasion, tearing and perforations. There is a startling paucity of basic knowledge on calcification mechanisms, as well as action of treatments that presumably mitigate calcification [40].

The goal of heart valve tissue engineering (HVTE) is to reestablish proper valve functions while allowing for slow regeneration of a new valve tissue. These two processes have to occur simultaneously without influencing each other or promoting thrombosis, coagulation or inflammation. Immediately upon implantation, the device needs to function properly, to withstand the harsh biomechanical regime of the aortic valve environment and induce left ventricular remodeling. While performing these functions, the device is expected to be perfectly "tuned" so that endothelial and interstitial cells slowly remodel the ECM without altering mechanical properties in the process. This is a challenging task, as the balance between MMPs and their inhibitors is very delicate [58].

Strategies for HVTE fall into several categories [61]. A first approach is implantation of decellularized valve matrices and relying on host cells to repopulate and remodel the scaffolds. This approach has been limited by the fact that scaffolds are not porous enough, cells do not readily infiltrate the scaffolds and human acellular valves are in short supply. Porcine acellular valves have been implanted in recent years in several clinics, but their use is currently limited due to early failure of several valves in clinical studies [62]. Some valves exhibited severe inflammation after only 2 days of implantation, leading to structural failure at 7 days and degeneration associated with severe foreign body reaction dominated by early neutrophils and macrophage infiltration and late lymphocyte reactions. These reactions were the result of incomplete cell removal and other manufacturing problems [62]. A second alternative would be to repopulate decellularized valve matrices with appropriate cell types in vitro before implantation. Recently we have shown that in vitro endothelialization of acellular porcine aortic valves is possible and that surface-seeded endothelial cells withstood bioreactor testing in pulmonary conditions for
3 weeks showing excellent retention and viability [63]. A third strategy involves assembly of synthetic biodegradable matrices populated by cells and bioreactor conditioning to express adequate properties before implantation [29, 64]. While this approach seems appealing, the polymeric matrices lack sufficient mechanical strength [65], develop fibrosis [64] and have not yet withstood the test of time under arterial pressures.

5.1. Prognosis. Tissue engineering of heart valves is one of the most daunting tasks in the field of regenerative medicine. Technologically, there are numerous hurdles to overcome before implantable living heart valves become a reality. Valvular scaffolds have to function immediately after implantation, endure significant mechanical stress, and also maintain stable matrix homeostasis while undergoing adaptation to altered hemodynamics and somatic growth. The presence of living interstitial cells and a continuous layer of endothelial cells covering the scaffolds would prove highly beneficial ensuring proper matrix homeostasis and protection from inflammation. Further studies are needed to make the dream of HVTE a reality.

\section{Vascular Tissue Engineering}

Current treatment options for vascular diseases, regardless of location in the vasculature, include balloon angioplasty, stent placement, graft bypass surgery, and use of pharmacological agents [66-70]. The total need for vascular grafts has been estimated to be more than 1.4 million in the USA alone [71]. These can be divided into three categories, in order of decreasing diameter. The large and medium caliber synthetic grafts are used in the thoracic and abdominal cavities with good long-term outcomes. Almost 1,200,000 small-caliber grafts $(<6 \mathrm{~mm})$ are used every year for vascular access, to relieve lower limb ischemia and for coronary bypass surgery. Autologous veins or arteries are the "gold standard" for replacement of small caliber arteries, but in 30-40\% of patients these are not available due to prior harvesting or preexisting conditions. In these latter cases synthetic grafts are used, but they provide poor outcomes, as 50\% of these will occlude within 5 years [72], potentially leading to amputation.

Tissue engineering vascular grafts (TEVGs) entails the growing of living vessels, by using cells and scaffolds, alone or in combinations. The functional requirements must include the following: nonthrombogenicity, adequate burst pressure and compliance, appropriate remodeling responses, and vasoactivity. Equally important is the propensity to elicit inflammation and to resist proinflammatory milieus when implanted in compromised patients. Traditionally, TEVGs have been constructed from both biological and synthetic scaffolds [73] with or without repopulation with autologous cells [72]. For these purposes, mesenchymal stem cells derived from a patient are purified, expanded, and exposed to specific biological cues to incite differentiation into desired cell types, and the TEVGs are then seeded with cells $[74,75]$. Cell-seeded grafts are then cultured in bioreactors to adapt 
to increasing loads and increase overall performance [76]. Excellent results have been reported in animal testing and also limited number of clinical trials [77]; however, the longterm efficacy of these grafts is still uncertain. Attaining a proper balance between scaffold degradation and remodeling is still under investigation. Rapid degradation is clearly detrimental and could have dramatic consequences. Slow degradation of scaffolds is a prerequisite for successful TEVGs; however, scaffold degradation products (collagen, elastin, fibronectin and laminin peptides) in synergism with TGF-beta and other cytokines may induce the differentiation of smooth muscle cells and fibroblasts into osteoblast-like cells and myofibroblasts, respectively [47, 78-80].

\subsection{Existing Inflammation: The Bane of Tissue-Engineered} Vascular Grafts. One of the most complicated aspects of designing a replacement for diseased tissue is incorporating measures which prevent the device from succumbing to the same fate as the diseased tissue it is replacing. The clinical experience with the transplantation of vascularized organs has uncovered several aspects useful for TEVGs. In heart transplants, it has been reported that as the duration of time after-implant increases, patient mortality also increases [81]. The culprit for this problem has been denoted as graft arterial disease (GAD) [82], characterized by intimal hyperplasia, the denudation of the medial layers, fibrosis of adventitial layers, and vasoconstriction [83]. GAD differs from atherosclerosis by not being related to fatty streak deposition and by developing circumferentially and not focally [84]. Shimizu and Mitchell [82] also reported that symptoms of ischemia are often masked in transplanted tissues due to the lack of innervation, only furthering the convolution of the disease. Therefore, even following successful organ transplantation and the necessary immunosuppression medication that ensues, GAD can arise and threaten the health of both the transplanted organ and the patient. Placing TEVGs into a compromised site would likely decrease the life of the vascular construct, especially due to the expression of chemokines that recruit inflammatory cells which are key to allograft rejection $[85,86]$. GAD has been reported to incite host ingrowth of endothelial cells and intimal smooth muscle-like cells, which are reported to be different from medial-smooth muscle cells [87], into the allograft vasculature [88-90]. On the upside, such cellular ingrowth properties could potentially be harnessed to further TE strategies for vascular constructs.

Atherosclerosis has similarities to GAD but ultimately differs in the involvement of lipoproteins and foam cells entrapped within the intima, which form the characteristic localized fatty streaks [23]. After several decades of research, we now know that atherosclerosis is a consequence of chronic inflammation [91] with elements of immune system activation [92]. The main questions that bioengineers have to address now are how to develop a TEVG that would be protected from atherosclerosis after implantation in an atherosclerotic prone patient? Will an endothelium that is healthy at implantation become dysfunctional under systemic insults, and, therefore, not be capable of preventing local inflammatory signals? Is control of systemic inflammation in fact the critical step to success? Regardless of the mechanisms perpetuating disease progression, studies into pathogenesis of atherosclerosis and other inflammatory diseases point to the activation of endothelial cells as the critical starting point for the disease $[23,93]$. Thus, knowledge of mechanisms of endothelium activation and its role in promoting inflammation would need to be incorporated into future TEVGs [23].

6.2. Prognosis. The field of vascular tissue engineering necessitates advances in cell seeding, cell ingrowth, elastin biosynthesis, and nutrient perfusion before any large clinical breakthroughs are to be expected. Scaffolds need to be altered so that they are less inflammatory and also that they resist and perform well in proinflammatory environments. For these objectives, testing TEVGs in healthy and pathological animal models would be useful. The ability of vascular grafts to fully remodel into de novo formed arteries still needs to be evaluated extensively.

\section{Myocardial Tissue Engineering}

Aside from replacements for ailing valves and devices used to occlude septal defects, applications for tissue engineering which deal solely with the myocardium are very limited. A major area of interest, however, is the restoration of cardiac muscle functionality in patients who have suffered an acute myocardial infarction (AMI) and are at risk for developing congestive heart failure (CHF) [94-97]. The acute inflammatory response which occurs subsequently to the death of ischemic cells is culpable for scar tissue development, which, in turn, is responsible for global remodeling of the heart and, ultimately, development of $\mathrm{CHF}$ $[24,94,96]$. This scenario is different from inflammation in heart valves or blood vessels because the post-AMI inflammatory response is a consequence of or response to, ischemia. Injury leads to the activation of inflammation, ECM degradation and MMPs and TGF secretion which in turn activate cardiac fibroblasts to initiate fibrosis [98100]. Clearly, vascular endothelium is the main barrier that controls tissue reactivity to cytokines and determines the outcome of tissue inflammation [101]. Tissue engineering approaches to the regeneration of the myocardium following AMI strive to attenuate the geometrical alterations (dilated or ischemic cardiomyopathy) which take place in the ventricles, concurrently slowing or halting progression towards $\mathrm{CHF}$ $[95,102]$.

The remainder of this section will discuss only efforts to regenerate the myocardium which involve the use of engineered heart tissue (EHT) [103] constructs, defined as consisting of a scaffold composed of synthetic material, natural material, or any combination thereof and at least one type of cell attached to the scaffold. Ideally, the EHT should be prevascularized, posses similar mechanical properties to ventricular myocardium, be contractile and integrate well within existing tissues. More importantly it should be 
noninflammatory and also resist the proinflammatory environment surrounding the infarcted area. We will specifically address several points which are relevant to the feasibility of using EHT to treat ischemic cardiomyopathy, including the interaction of EHT with existing scar tissue, the host response to the construct and the challenges of implanting EHT in patients with poor cardiovascular health or other preexisting conditions.

7.1. Complications from Existing Scar Tissue. The inflammatory response following AMI occurs rapidly. It is estimated that necrosis of cardiomyocytes begins 20-30 minutes after the initial blockage of the coronary vasculature [104]. The current gold standard in clinical care after-AMI is percutaneous coronary intervention (PCI), a transcatheter procedure wherein a balloon is used to dilate the vessel lumen immediately adjacent to the site of thrombotic occlusion, thus restoring flow to the downstream tissues [105]. Currently, chest pain centers and cardiac care units have set a benchmark "door-to-balloon" time of 90 minutes in order to limit the extent of damage produced by AMI [106, 107]. While minimizing door-to-balloon time is critical, it is difficult to imagine achieving the reperfusion of the ischemic tissue before cardiomyocyte necrosis sets in.

When necrosis occurs, so will inflammation. And with a lack of intrinsic regenerative capability in the myocardium, the necrotic core will be completely remodeled into fibrous tissue in 2-6 weeks after-AMI [97, 108, 109]. The implications of this abnormal remodeling are twofold: (a) tissueengineering strategies currently hold the most promise for regeneration of the myocardium because they introduce new, viable cells into an environment that is not conducive to regeneration on its own [94]; (b) tissue engineered constructs will have to be placed over or adjacent to scar tissue unless the construct is implanted immediately following AMI (before scar tissue can be formed) or the scar tissue is surgically resected prior to the implantation of the construct [102]. In order to implant a construct immediately after AMI, it would need to be ready before the event, in an "off the shelf" fashion. This possibility is precluded by complications that arise due to autologous cell sourcing and the need for in vitro cell proliferation, seeding, and preconditioning in a bioreactor. Such a process could take several weeks, if not months, to complete and could not benefit the patient immediately unless the AMI was predicted $[95,102,108]$.

Whether or not grafting EHT [103] directly to the fibrotic scar inhibits proper adherence, and the perfusion of the graft with the host tissue through an inflammatory response is unclear. A number of studies have reported positive outcomes when implanting EHT on top of infarct scars created in animal models [110-112]. When EHT constructs are not placed directly on the epicardium over the scar, but rather at the endocardial surface (by means of a Dor procedure) [113] or in a depression made by the resection of the scar tissue, solid engraftment has been repeatedly achieved [114-116]. These opposing implantation methods will need to be compared for efficacy by performing both with a single type of EHT, then perhaps the difference, if one exists, can be elucidated.

Equally conflicting results were reported by two groups who implanted "prevascularized" EHT in a nude rat model (without infarct). One group reported that their construct integrated well, forming microvasculature which perfused with the host's and resembled viable myocardium [117], while another group observed fibrotic encapsulation of their EHT graft. Ironically, the latter occurred in rats which were on an immunosuppression regimen [97]. What is clear, however, is that cell-free constructs composed of synthetic material can be at risk for poor performance when implanted in or adjacent to the myocardium, possibly due to inflammation. Stuckey et al. reported that patches of titanium oxide-reinforced poly(ethylene terephthalate)/dimer fatty acid implanted onto infarcted rat hearts produced extensive necrosis in the adjacent healthy host tissue, most likely due to micromotions between the graft and host caused by compliance mismatch [94]. Similarly, another group reported extensive foreign body response, thrombus formation, necrosis, and calcification associated with an ePTFE graft that was implanted on the endocardial surface of the infarcted heart in a pig model [115]. It is evident that control of inflammation in EHT is of essence for the success of implanted scaffolds and that this might be enhanced by the preexisting "inflamed" status of the ischemic myocardium.

7.2. Characterizing the Host Response. Positive outcomes in designing EHT are measured by criteria such as the attenuation of ventricular wall remodeling or dilation, perfusion of the graft by the host vasculature, electrical coupling of the cardiomyocytes in the graft with those of the host (via the formation of connexins or gap junctions), and the avoidance of inflammation, encapsulation, or calcification [95, 102, 108]. Tissue engineering for this specific application is still in its early stages. Most implantation studies have not been run for longer than 3 months and have focused on establishing proof of principle rather than studying the longterm performance of EHT constructs [110-112, 114-116]. As such, there is very little data available in regards to chronic inflammatory responses or calcification with which to make a prognosis for the long term viability of these constructs.

The first ever clinical trial (MAGNUM-trial) involving an EHT construct was begun in 2003 and used autologous bone marrow cells (BMC) seeded onto a 3D collagen type I scaffold [118]. The construct was implanted over the epicardial surface of the postinfarct scar in a group of 10 patients while they were undergoing coronary artery bypass grafting surgery. Followup at 10 months indicated prevention of cardiac remodeling and thickening of the scar (via cell ingrowth) with functional, healthy tissue. These were assessed using echocardiography and single-photon emission computed tomography [119]. There were no indications of immune or inflammatory reactions, no arrhythmias, and the EHT appeared to integrate well with the host tissue. It is important to note that this particular EHT construct was not intended to be functional, meaning it was neither contractile 
nor vascularized [102]. Its mechanism of action was one of providing "passive" support, and the collagen matrix most likely acting as a stable microenvironment from which the BMC could secrete paracrine signals to the healthy cells in the peri-infarct tissue. A construct of this type, implanted in this fashion, would not be at risk for developing either GAD or atherosclerosis, because the cells were autologous and the neovasculature formed within it would not consist of vessels large enough to be afflicted with such conditions [120, 121]. Calcification, however, might develop in this construct because type I collagen is known to predispose to forming nucleation sites for calcium deposits [122]. Furthermore, the mechanical stresses and deformations imposed on the construct via the continuous, repetitive contraction of the heart might accelerate mineralization [122]. It is noted that the authors of the MAGNUM trial make no mention of calcification being observed in the followup, especially since echocardiography can be used to image calcium [123]. In the case of the MAGNUM trial, it is probable that more time must elapse before evidence of the activity of the typical culprits in the failure of tissue-engineered constructs emerges.

7.3. Engineered Heart Tissue in the Compromised Patient. The vast majority of patients who could benefit from a clinically feasible and cost-effective EHT have some form of cardiovascular disease [95]. When developing EHT, researchers should rid themselves of the illusion that potential candidates for their therapy will be a tabula rasa of health. Rather, they should prepare EHT constructs to withstand a hostile, diseased environment. In the MAGNUM trial discussed earlier, for example, 11 of the 20 participants were hypertensive, 13 were hypercholesterolemic, 7 were diabetic, and 10 were smokers [118].

In terms of inflammatory processes, a few of the most threatening conditions to the long-term viability of EHT are atherosclerosis, GAD, and arteriolosclerosis. Atherosclerosis is the most well known of these diseases, as it is implicated in having a causal role in both CHD and peripheral artery disease. Characteristic atheromatous plaques or lesions also indicate the possibility of tissue calcification [120, 124, 125]. GAD has been observed to occur predominantly in implanted allografts, which contain immunogenic cell constituents [121]. EHT constructs, however, should ideally consist of nonimmunogenic ECM proteins seeded with autologous cells, rendering them free from concerns about GAD or transplant vasculopathy [121]. Finally, arteriolosclerosis, or the thickening of the intimal layer in arterioles due to hyperplasia or hyaline matrix deposition, could occur in EHT [120]. EHT grafts implanted in hypertensive or diabetic patients would be at an increased risk of contracting arteriolosclerosis because these risk factors are closely interrelated [120]. Both atherosclerosis and arteriolosclerosis could have a major impact upon the viability of EHT constructs, because such constructs will most likely contain a preformed or preserved vascular network that will stenose or occlude if these diseases are present, leading to ischemia and necrosis of the cells in the construct.
7.4. Prognosis. It is difficult to make conjectures about the ultimate success or failure of EHT constructs. Given the requirements placed upon them and the mechanisms or processes that might be at work in vivo, producing an EHT construct that remains viable for some time will be a challenging task. In the future, more clinical trials of EHT constructs need to be conducted so that a larger data set can be formed and the efficacy of their outcomes assessed. The pathways which mediate the inflammatory processes of atherosclerosis and arteriolosclerosis, the fibrous remodeling process, and the unfriendly microenvironment of patients with these conditions need to be elucidated so that drug discovery efforts can generate new prophylactic drugs. While such drugs could reduce the patient population in need of EHT treatments, the current demand is so great that the need for them would still be obviated [95]. Until such drugs are available, however, EHT needs to be developed so as to withstand the hostile environs in patients compromised by diseases. Short of this, the best case scenario is that patients receiving EHT grafts will cease or seek treatment for risky behaviors such as smoking, hypertension, hypercholesterolemia, or diabetes and initiate beneficial behaviors like engaging in physical activity or adjusting dietary intake and nutrition. Sadly, inflammatory processes are self perpetuating in patients afflicted with cardiovascular disease. The progression to $\mathrm{CHF}$ can begin with one chronic inflammatory condition which begets another acute inflammatory response, constituting a chain of causality. If researchers working to develop EHT can overcome the inherent challenges associated with it, they can alleviate the devastation CVD causes so many patients and their families throughout the world.

\section{Final Remarks}

CVTE holds great potential to solve some of the biggest current health issues. The prospects of using scaffolds, cells, and chemical or mechanical stimuli to create functional tissues such as valves, arteries and myocardium are hugely exciting as we attain "magical powers" previously unimaginable. After an initial period of hype and hope, we are now closer to clinical application of CVTE; however, we need to increase our control over inflammation and its clinical consequences. One approach could involve making scaffolds from carefully screened, intact molecules (synthetic of biologic), and repopulating the scaffolds with interstitial cells and endothelial cells in vitro. This should be followed by conditioning the constructs in bioreactors until cells "gain control" over the remodeling process and the constructs show clear signs of regeneration. Only then would tissue engineering products be ready for implantation in the targeted dynamic cardiovascular sites. While we continue to trek towards the unknown at warp speed, we need to focus more on the endothelium, the "final frontier". This will eventually determine whether novel CVTE devices will successfully help thousands of patients in need of tissue regeneration and repair. 


\section{References}

[1] G. Orlando, P. Baptista, M. Birchall et al., "Regenerative medicine as applied to solid organ transplantation: current status and future challenges," Transplant International, vol. 24, pp. 223-232, 2011.

[2] C. Landis, "Why the inflammatory response is important to the cardiac surgical patient," Journal of Extra-Corporeal Technology, vol. 39, no. 4, pp. 281-284, 2007.

[3] D. F. Williams, "On the mechanisms of biocompatibility," Biomaterials, vol. 29, no. 20, pp. 2941-2953, 2008.

[4] D. Williams, "Metastable biocompatibility: a new approach," Medical Device Technology, vol. 18, no. 8, pp. 10-11, 2007.

[5] A. E. Trantina-Yates, P. Human, M. Bracher, and P. Zilla, "Mitigation of bioprosthetic heart valve degeneration through biocompatibility: in vitro versus spontaneous endothelialization," Biomaterials, vol. 22, no. 13, pp. 18371846, 2001.

[6] S. L. Robbins, V. Kumar, and R. S. Cotran, Robbins and Cotran Pathologic Basis Of Disease, Saunders, Philadelphia, $\mathrm{Pa}, \mathrm{USA}, 2010$.

[7] P. Libby and P. M. Ridker, "Inflammation and atherothrombosis. from population biology and bench research to clinical practice," Journal of the American College of Cardiology, vol. 48, no. 9, pp. A33-A46, 2006.

[8] J. W. Mier, E. P. Brandon, P. Libby, M. W. Janicka, and F. R. Aronson, "Activated endothelial cells resist lymphokineactivated killer cell-mediated injury. possible role of induced cytokines in limiting capilarry leak during IL-2 therapy," Journal of Immunology, vol. 143, no. 7, pp. 2407-2414, 1989.

[9] C. J. Diskin, "Novel insights into the pathobiology of the vascular access-do they translate into improved care?" Blood Purification, vol. 29, no. 2, pp. 216-229, 2010.

[10] K. A. Cieslik, G. E. Taffet, S. Carlson, J. Hermosillo, J. Trial, and M. L. Entman, "Immune-inflammatory dysregulation modulates the incidence of progressive fibrosis and diastolic stiffness in the aging heart," Journal of Molecular and Cellular Cardiology, vol. 50, no. 1, pp. 248-256, 2011.

[11] F. J. Schoen, "Heart valve tissue engineering: quo vadis?" Current Opinion in Biotechnology, vol. 39, no. 1, pp. 205-222, 2011.

[12] M. Dahm, W. D. Lyman, A. B. Schwell, S. M. Factor, and R. W. Frater, "Immunogenicity of glutaraldehyde-tanned bovine pericardium," Journal of Thoracic and Cardiovascular Surgery, vol. 99, no. 6, pp. 1082-1090, 1990.

[13] J. E. Feig, S. Parathath, J. X. Rong et al., "Reversal of hyperlipidemia with a genetic switch favorably affects the content and inflammatory state of macrophages in atherosclerotic plaques," Circulation, vol. 123, pp. 989-998, 2011.

[14] S. Devaraj and I. Jialal, "C-reactive protein polarizes human macrophages to an $\mathrm{m} 1$ phenotype and inhibits transformation to the $\mathrm{m} 2$ phenotype," Arteriosclerosis Thrombosis and Vascular Biology. In press.

[15] J. Mikita, N. Dubourdieu-Cassagno, M. S. Deloire et al., "Altered $\mathrm{m} 1 / \mathrm{m} 2$ activation patterns of monocytes in severe relapsing experimental rat model of multiple sclerosis. amelioration of clinical status by $\mathrm{m} 2$ activated monocyte administration," Multiple Sclerosis, vol. 17, pp. 2-15, 2011.

[16] S. F. Badylak, J. E. Valentin, A. K. Ravindra, G. P. McCabe, and A. M. Stewart-Akers, "Macrophage phenotype as a determinant of biologic scaffold remodeling," Tissue Engineering Part A, vol. 14, no. 11, pp. 1835-1842, 2008.
[17] M. B. Nourse, D. E. Halpin, M. Scatena et al., "Vegf induces differentiation of functional endothelium from human embryonic stem cells: implications for tissue engineering," Arteriosclerosis Thrombosis and Vascular Biology, vol. 30, no. 1, pp. 80-89, 2010.

[18] J. R. Lindner, "Contrast ultrasound molecular imaging of inflammation in cardiovascular disease," Cardiovascular Research, vol. 84, no. 2, pp. 182-189, 2009.

[19] S. Kaul and J. R. Lindner, "Visualizing coronary atherosclerosis in vivo: thinking Big, imaging Small," Journal of the American College of Cardiology, vol. 43, no. 3, pp. 461-463, 2004.

[20] S. A. Wickline, A. M. Neubauer, P. Winter, S. Caruthers, and G. Lanza, "Applications of nanotechnology to atherosclerosis, thrombosis, and vascular biology," Arteriosclerosis Thrombosis and Vascular Biology, vol. 26, no. 3, pp. 435-441, 2006.

[21] F. A. Jaffer, C. Vinegoni, M. C. John et al., "Real-time catheter molecular sensing of inflammation in proteolytically active atherosclerosis," Circulation, vol. 118, no. 18, pp. 1802-1809, 2008.

[22] B. A. Kaufmann, J. M. Sanders, C. Davis et al., "Molecular imaging of inflammation in atherosclerosis with targeted ultrasound detection of vascular cell adhesion molecule-1," Circulation, vol. 116, no. 3, pp. 276-284, 2007.

[23] P. Libby, P. M. Ridker, and G. K. Hansson, "Inflammation in atherosclerosis," Journal of the American College of Cardiology, vol. 54, no. 23, pp. 2129-2138, 2009.

[24] J. Danesh, J. G. Wheeler, G. M. Hirschfield et al., "C-reactive protein and other circulating markers of inflammation in the prediction of coronary heart disease," New England Journal of Medicine, vol. 350, no. 14, pp. 1387-1397, 2004.

[25] R. J. Glynn, J. G. MacFadyen, and P. M. Ridker, "Tracking of high-sensitivity c-reactive protein after an initially elevated concentration: the jupiter study," Clinical Chemistry, vol. 55, no. 2, pp. 305-312, 2009.

[26] P. M. Ridker, "C-reactive protein and the prediction of cardiovascular events among those at intermediate risk. moving an inflammatory hypothesis toward consensus," Journal of the American College of Cardiology, vol. 49, no. 21, pp. 2129-2138, 2007.

[27] P. M. Ridker, C. P. Cannon, D. Morrow et al., "C-reactive protein levels and outcomes after statin therapy," New England Journal of Medicine, vol. 352, no. 1, pp. 20-28, 2005.

[28] Y. Naito, T. Shinoka, D. Duncan et al., "Vascular tissue engineering: towards the next generation vascular grafts," Advanced Drug Delivery Reviews, vol. 63, no. 4-5, pp. 312323, 2011.

[29] B. Weber, M. Y. Emmert, R. Schoenauer, C. Brokopp, L. Baumgartner, and S. P. Hoerstrup, "Tissue engineering on matrix: future of autologous tissue replacement," Seminars in Immunopathology, 2011.

[30] H. M. Nugent and E. R. Edelman, "Tissue engineering therapy for cardiovascular disease," Circulation Research, vol. 92, no. 10, pp. 1068-1078, 2003.

[31] S. S. Apte, A. Paul, S. Prakash, and D. Shum-Tim, "Current developments in the tissue engineering of autologous heart valves: moving towards clinical use," Future Cardiology, vol. 7, no. 1, pp. 77-97, 2011.

[32] A. G. Mikos, S. W. Herring, P. Ochareon et al., "Engineering complex tissues," Tissue Engineering, vol. 12, no. 12, pp. 3307-3339, 2006.

[33] E. Rabkin and F. J. Schoen, "Cardiovascular tissue engineering," Cardiovascular Pathology, vol. 11, no. 6, pp. 305-317, 2002. 
[34] S. F. Badylak, "The extracellular matrix as a biologic scaffold material," Biomaterials, vol. 28, no. 25, pp. 3587-3593, 2007.

[35] M. T. Kasimir, E. Rieder, G. Seebacher, E. Wolner, G. Weigel, and P. Simon, "Presence and elimination of the xenoantigen gal (alpha1, 3) gal in tissue-engineered heart valves," Tissue Engineering, vol. 11, no. 7-8, pp. 1274-1280, 2005.

[36] S. F. Badylak, "Xenogeneic extracellular matrix as a scaffold for tissue reconstruction," Transplant Immunology, vol. 12, no. 3-4, pp. 367-377, 2004.

[37] M. E. Tedder, J. Liao, B. Weed et al., "Stabilized collagen scaffolds for heart valve tissue engineering," Tissue Engineering Part A, vol. 15, no. 6, pp. 1257-1268, 2009.

[38] T. H. Chuang, C. Stabler, A. Simionescu, and D. T. Simionescu, "Polyphenol-stabilized tubular elastin scaffolds for tissue engineered vascular grafts," Tissue Engineering Part A, vol. 15, no. 10, pp. 2837-2851, 2009.

[39] D. T. Simionescu, Q. Lu, Y. Song et al., "Biocompatibility and remodeling potential of pure arterial elastin and collagen scaffolds," Biomaterials, vol. 27, no. 5, pp. 702-713, 2006.

[40] D. T. Simionescu, "Prevention of calcification in bioprosthetic heart valves: challenges and perspectives," Expert Opinion on Biological Therapy, vol. 4, no. 12, pp. 1971-1985, 2004.

[41] A. Simionescu, D. Simionescu, and R. Deac, "Lysineenhanced glutaraldehyde crosslinking of collagenous biomaterials," Journal of Biomedical Materials Research, vol. 25, no. 12, pp. 1495-1506, 1991.

[42] D. Bezuidenhout, A. Oosthuysen, P. Human, C. Weissenstein, and P. Zilla, "The effects of cross-link density and chemistry on the calcification potential of diamine-extended glutaraldehyde-fixed bioprosthetic heart-valve materials," Biotechnology and Applied Biochemistry, vol. 54, pp. 133-140, 2009.

[43] A. E. Trantina-Yates, P. Human, and P. Zilla, "Detoxification on top of enhanced, diamine-extended glutaraldehyde fixation significantly reduces bioprosthetic root calcification in the sheep model," Journal of Heart Valve Disease, vol. 12, no. 1, pp. 93-101, 2003.

[44] P. Huma, D. Bezuidenhout, M. Torrianni, M. Hendriks, and P. Zilla, "Optimization of diamine bridges in glutaraldehyde treated bioprosthetic aortic wall tissue," Biomaterials, vol. 23, no. 10, pp. 2099-2103, 2002.

[45] P. Zilla, D. Bezuidenhout, C. Weissenstein, A. Van Der Walt, and P. Human, "Diamine extension of glutaraldehyde crosslinks mitigates bioprosthetic aortic wall calcification in the sheep model," Journal of Biomedical Materials Research, vol. 56, no. 1, pp. 56-64, 2001.

[46] J. S. Lee, D. M. Basalyga, A. Simionescu, J. C. Isenburg, D. T. Simionescu, and N. R. Vyavahare, "Elastin calcification in the rat subdermal model is accompanied by up-regulation of degradative and osteogenic cellular responses," American Journal of Pathology, vol. 168, no. 2, pp. 490-498, 2006.

[47] A. Simionescu, K. Philips, and N. Vyavahare, "Elastinderived peptides and tgf-betal induce osteogenic responses in smooth muscle cells," Biochemical and Biophysical Research Communications, vol. 334, no. 2, pp. 524-532, 2005.

[48] D. M. Basalyga, D. T. Simionescu, W. Xiong, B. T. Baxter, B. C. Starcher, and N. R. Vyavahare, "Elastin degradation and calcification in an abdominal aorta injury model: role of matrix metalloproteinases," Circulation, vol. 110, no. 22, pp. 3480-3487, 2004.
[49] J. C. Isenburg, D. T. Simionescu, and N. R. Vyavahare, "Tannic acid treatment enhances biostability and reduces calcification of glutaraldehyde fixed aortic wall," Biomaterials, vol. 26, no. 11, pp. 1237-1245, 2005.

[50] J. C. Isenburg, N. V. Karamchandani, D. T. Simionescu, and N. R. Vyavahare, "Structural requirements for stabilization of vascular elastin by polyphenolic tannins," Biomaterials, vol. 27, no. 19, pp. 3645-3651, 2006.

[51] J. C. Isenburg, D. T. Simionescu, B. C. Starcher, and N. R. Vyavahare, "Elastin stabilization for treatment of abdominal aortic aneurysms," Circulation, vol. 115, no. 13, pp. 17291737, 2007.

[52] S. K. Singh, N. D. Desai, S. D. Petroff et al., "The impact of diabetic status on coronary artery bypass graft patency: insights from the radial artery patency study," Circulation, vol. 118, no. 14, pp. S222-S225, 2008.

[53] C. Marchand, F. Heim, and B. A. Durand, "A novel stent for percutaneous heart valve implantation: first in vitro results," Journal of Biomechanical Engineering, vol. 132, no. 5, Article ID 054502, 2010.

[54] G. Soldani, P. Losi, M. Bernabei et al., "Long term performance of small-diameter vascular grafts made of a poly(ether)urethane-polydimethylsiloxane semiinterpenetrating polymeric network," Biomaterials, vol. 31, no. 9, pp. 2592-2605, 2010.

[55] J. G. Meinhart, M. Deutsch, T. Fischlein, N. Howanietz, A. Fröschl, and P. Zilla, "Clinical autologous in vitro endothelialization of 153 infrainguinal eptfe grafts," Annals of Thoracic Surgery, vol. 71, no. 5, pp. S327-S331, 2001.

[56] P. Zilla, D. Bezuidenhout, and P. Human, "Prosthetic vascular grafts: wrong models, wrong questions and no healing," Biomaterials, vol. 28, no. 34, pp. 5009-5027, 2007.

[57] B. Jian, N. Narula, Q. Y. Li, E. R. Mohler, and R. J. Levy, "Progression of aortic valve stenosis: Tgf-betal is present in calcified aortic valve cusps and promotes aortic valve interstitial cell calcification via apoptosis," Annals of Thoracic Surgery, vol. 75, no. 2, pp. 457-465, 2003.

[58] F. J. Schoen, "Evolving concepts of cardiac valve dynamics: the continuum of development, functional structure, pathobiology, and tissue engineering," Circulation, vol. 118, no. 18, pp. 1864-1880, 2008.

[59] P. Zilla, J. Brink, P. Human, and D. Bezuidenhout, "Prosthetic heart valves: catering for the few," Biomaterials, vol. 29, no. 4, pp. 385-406, 2008.

[60] M. R. Abboud, J. Cure, S. Granger et al., "Magnetic resonance angiography in children with sickle cell disease and abnormal transcranial Doppler ultrasonography findings enrolled in the stop study," Blood, vol. 103, no. 7, pp. 2822-2826, 2004.

[61] K. Mendelson and F. J. Schoen, "Heart valve tissue engineering: concepts, approaches, progress, and challenges," Annals of Biomedical Engineering, vol. 34, no. 12, pp. 1799-1819, 2006.

[62] P. Simon, M. T. Kasimir, G. Seebacher et al., "Early failure of the tissue engineered porcine heart valve synergraft in pediatric patients," European Journal of Cardio-Thoracic Surgery, vol. 23, no. 6, pp. 1002-1006, 2003.

[63] L. N. Sierad, A. Simionescu, C. Albers et al., "Design and testing of a pulsatile conditioning system for dynamic endothelialization of polyphenol-stabilized tissue engineered heart valves," Cardiovascular Engineering and Technology, pp. 138-153, 2010. 
[64] D. Schmidt, P. E. Dijkman, A. Driessen-Mol et al., "Minimally-invasive implantation of living tissue engineered heart valves: a comprehensive approach from autologous vascular cells to stem cells," Journal of the American College of Cardiology, vol. 56, no. 6, pp. 510-520, 2010.

[65] P. S. Robinson, S. L. Johnson, M. C. Evans, V. H. Barocas, and R. T. Tranquillo, "Functional tissue-engineered valves from cell-remodeled fibrin with commissural alignment of cellproduced collagen," Tissue Engineering Part A, vol. 14, no. 1, pp. 83-95, 2008.

[66] P. M. Ridker, E. Danielson, F. A. H. Fonseca et al., "Rosuvastatin to prevent vascular events in men and women with elevated c-reactive protein," New England Journal of Medicine, vol. 359, no. 21, pp. 2195-2207, 2008.

[67] J. Chlupác, E. Filová, and L. Bacáková, "Blood vessel replacement: 50 years of development and tissue engineering paradigms in vascular surgery," Physiological Research, vol. 58, pp. S119-S139, 2009.

[68] D. Tsetis, "Endovascular treatment of complications of femoral arterial access," CardioVascular and Interventional Radiology, vol. 33, no. 3, pp. 457-468, 2010.

[69] M. Thatipelli and S. Misra, "Endovascular intervention for renal artery stenosis," Abdominal Imaging, no. 35, pp. 612621, 2009.

[70] I. I. Galaria, S. M. Surowiec, J. M. Rhodes et al., "Percutaneous and open renal revascularizations have equivalent long-term functional outcomes," Annals of Vascular Surgery, vol. 19, no. 2, pp. 218-228, 2005.

[71] L. E. Niklason and R. S. Langer, "Advances in tissue engineering of blood vessels and other tissues," Transplant Immunology, vol. 5, no. 4, pp. 303-306, 1997.

[72] F. J. Veith, S. K. Gupta, E. Ascer et al., "Six-year prospective multicenter randomized comparison of autologous saphenous vein and expanded polytetrafluoroethylene grafts in infrainguinal arterial reconstructions," Journal of Vascular Surgery, vol. 3, pp. 104-114, 1986.

[73] A. Patel, B. Fine, M. Sandig, and K. Mequanint, "Elastin biosynthesis: the missing link in tissue-engineered blood vessels," Cardiovascular Research, vol. 71, no. 1, pp. 40-49, 2006.

[74] K. M. Sales, H. J. Salacinski, N. Alobaid, M. Mikhail, V. Balakrishnan, and A. M. Seifalian, "Advancing vascular tissue engineering: the role of stem cell technology," Trends in Biotechnology, vol. 23, no. 9, pp. 461-467, 2005.

[75] S. Kim and H. V. Recum, "Endothelial stem cells and precursors for tissue engineering: cell source, differentiation, selection, and application," Tissue Engineering Part B, vol. 14, no. 1, pp. 133-147, 2008.

[76] C. Williams and T. Wick, "Perfusion bioreactor for small diameter tissue-engineered arteries," Tissue Engineering, vol. 10, no. 5-6, pp. 930-941, 2004.

[77] M. Desai, A. M. Seifalian, and G. Hamilton, "Role of prosthetic conduits in coronary artery bypass grafting," European Journal of Cardio-Thoracic Surgery. In press.

[78] A. G. Arroyo and M. L. Iruela-Arispe, "Extracellular matrix, inflammation, and the angiogenic response," Cardiovascular Research, vol. 86, no. 2, pp. 226-235, 2010.

[79] G. S. Schultz and A. Wysocki, "Interactions between extracellular matrix and growth factors in wound healing," Wound Repair and Regeneration, vol. 17, no. 2, pp. 153-162, 2009.

[80] F. Antonicelli, G. Bellon, L. Debelle, and W. Hornebeck, "Elastin-elastases and inflamm-aging," Current Topics in Developmental Biology, vol. 79, pp. 99-155, 2007.
[81] P. Libby and J. S. Pober, "Chronic rejection," Immunity, vol. 14, no. 4, pp. 387-397, 2001.

[82] K. Shimizu and R. N. Mitchell, "The role of chemokines in transplant graft arterial disease," Arteriosclerosis, Thrombosis, and Vascular Biology, vol. 28, no. 11, pp. 1937-1949, 2008.

[83] R. N. Mitchell and P. Libby, "Vascular remodeling in transplant vasculopathy," Circulation Research, vol. 100, no. 7, pp. 967-978, 2007.

[84] A. S. Neish, E. Loh, and F. J. Schoen, "Myocardial changes in cardiac transplant-associated coronary arteriosclerosis: potential for timely diagnosis," Journal of the American College of Cardiology, vol. 19, no. 3, pp. 586-592, 1992.

[85] J. J. Yun, M. P. Fischbein, H. Laks et al., "Early and late chemokine production correlates with cellular recruitment in cardiac allograft vasculopathy," Transplantation, vol. 69, no. 12, pp. 2515-2524, 2000.

[86] E. Di Carlo, T. D’Antuono, S. Contento, M. Di Nicola, E. Ballone, and C. Sorrentino, "Quilty effect has the features of lymphoid neogenesis and shares cxcl13-cxcr5 pathway with recurrent acute cardiac rejections," American Journal of Transplantation, vol. 7, no. 1, pp. 201-210, 2007.

[87] G. K. Owens, "Regulation of differentiation of vascular smooth muscle cells," Physiological Reviews, vol. 75, no. 3, pp. 487-517, 1995.

[88] K. Shimizu and R. N. Mitchell, "Chemokine-mediated recruitment of inflammatory and smooth muscle cells in transplant-associated arteriosclerosis," Current Opinion in Organ Transplantation, vol. 8, no. 1, pp. 55-63, 2003.

[89] K. Horiguchi, S. Kitagawa-Sakakida, Y. Sawa et al., "Selective chemokine and receptor gene expressions in allografts that develop transplant vasculopathy," Journal of Heart and Lung Transplantation, vol. 21, no. 10, pp. 1090-1100, 2002.

[90] D. X. Zhao, Y. Hu, G. G. Miller, A. D. Luster, R. N. Mitchell, and P. Libby, "Differential expression of the ifngamma-inducible cxcr3-binding chemokines, ifn-inducible protein 10, monokine induced by ifn, and association with cardiac allograft vasculopathy and acute rejection," Journal of Immunology, vol. 169, no. 3, pp. 1556-1560, 2002.

[91] P. Libby and G. K. Hansson, "Involvement of the immune system in human atherogenesis: current knowledge and unanswered questions," Laboratory Investigation, vol. 64, no. 1, pp. 5-15, 1991.

[92] G. K. Hansson and P. Libby, "The immune response in atherosclerosis: a double-edged sword," Nature Reviews Immunology, vol. 6, no. 7, pp. 508-519, 2006.

[93] J. J. Fuster, P. Fernandez, H. Gonzalez-Navarro, C. Silvestre, Y. N. A. Nahbah, and V. Andres, "Control of cell proliferation in atherosclerosis-insights from animal models and human studies," Cardiovascular Research, vol. 86, pp. 254-264, 2010.

[94] D. J. Stuckey, H. Ishii, Q. Z. Chen et al., "Magnetic resonance imaging evaluation of remodeling by cardiac elastomeric tissue scaffold biomaterials in a rat model of myocardial infarction," Tissue Engineering Part A, vol. 16, no. 11, pp. 3395-3402, 2010.

[95] H. Jawad, N. N. Ali, A. R. Lyon, Q. Z. Chen, S. E. Harding, and A. R. Boccaccini, "Myocardial tissue engineering: a review," Journal of Tissue Engineering and Regenerative Medicine, vol. 1, no. 5, pp. 327-342, 2007.

[96] H. K. Graham, M. Horn, and A. W. Trafford, "Extracellular matrix profiles in the progression to heart failure: european young physiologists symposium keynote lecture-bratislava 2007," Acta Physiologica, vol. 194, no. 1, pp. 3-21, 2008. 
[97] A. Lesman, M. Habib, O. Caspi et al., "Transplantation of a tissue-engineered human vascularized cardiac muscle," Tissue Engineering Part A, vol. 16, no. 1, pp. 115-125, 2010.

[98] M. Behnes, U. Hoffmann, S. Lang et al., "Transforming growth factor beta 1 (tgf-beta 1) in atrial fibrillation and acute congestive heart failure," Clinical Research in Cardiology, vol. 100, no. 4, pp. 335-342, 2010.

[99] T. K. Borg and R. Markwald, "Periostin: more than just an adhesion molecule," Circulation Research, vol. 101, no. 3, pp. 230-231, 2007.

[100] J. W. Holmes, T. K. Borg, and J. W. Covell, "Structure and mechanics of healing myocardial infarcts," Annual Review of Biomedical Engineering, vol. 7, pp. 223-253, 2005.

[101] S. Kofler, T. Nickel, and M. Weis, "Role of cytokines in cardiovascular diseases: a focus on endothelial responses to inflammation," Clinical Science, vol. 108, no. 3, pp. 205-213, 2005.

[102] W. H. Zimmermann, "Remuscularizing failing hearts with tissue engineered myocardium," Antioxidants and Redox Signaling, vol. 11, no. 8, pp. 2011-2023, 2009.

[103] R. I. Mehta, M. C. Fishbein, O. E. Solis et al., "Intravascular polymer material after coil embolization of a giant cerebral aneurysm," Human Pathology, vol. 40, no. 12, pp. 1803-1807, 2009.

[104] N. Jowett, D. Thompson, and R. Boyle, Comprehensive Coronary Care, Baillière Tindall, 2007.

[105] G. Esposito, S. Dellegrottaglie, and M. Chiariello, “The extent of irreversible myocardial damage and the potential for left ventricular repair after primary percutaneous coronary intervention," American Heart Journal, vol. 160, no. 6, pp. S4S10, 2010.

[106] J. C. Blankenship, T. D. Scott, K. A. Skelding et al., "Doorto-balloon times under $90 \mathrm{~min}$ can be routinely achieved for patients transferred for st-segment elevation myocardial infarction percutaneous coronary intervention in a rural setting," Journal of the American College of Cardiology, vol. 57, pp. 272-279, 2011.

[107] K. Nishida, S. K. Hirota, T. B. Seto et al., "Quality measure study: progress in reducing the door-to-balloon time in patients with st-segment elevation myocardial infarction," Hawaii Medical Journal, vol. 69, pp. 242-246, 2010.

[108] F. Wang and J. Guan, "Cellular cardiomyoplasty and cardiac tissue engineering for myocardial therapy," Advanced Drug Delivery Reviews, vol. 62, no. 7-8, pp. 784-797, 2010.

[109] K. T. Weber, "Cardiac interstitium in health and disease: the fibrillar collagen network," Journal of the American College of Cardiology, vol. 13, no. 7, pp. 1637-1652, 1989.

[110] W. H. Zimmermann, I. Melnychenko, G. Wasmeier et al., "Engineered heart tissue grafts improve systolic and diastolic function in infarcted rat hearts," Nature Medicine, vol. 12, no. 4, pp. 452-458, 2006.

[111] H. Piao, J. S. Kwon, S. Piao et al., "Effects of cardiac patches engineered with bone marrow-derived mononuclear cells and pgcl scaffolds in a rat myocardial infarction model," Biomaterials, vol. 28, no. 4, pp. 641-649, 2007.

[112] Q. Ke, Y. Yang, J. S. Rana, Y. Chen, J. P. Morgan, and Y. F. Xiao, "Embryonic stem cells cultured in biodegradable scaffold repair infarcted myocardium in mice," Sheng Li Xue Bao, vol. 57, pp. 673-681, 2005.

[113] M. Di Donato, M. Sabatier, V. Dor et al., "Effects of the dor procedure on left ventricular dimension and shape and geometric correlates of mitral regurgitation one year after surgery," Journal of Thoracic and Cardiovascular Surgery, vol. 121, no. 1, pp. 91-96, 2001.
[114] A. S. Krupnick, D. Kreisel, F. H. Engels et al., "A novel small animal model of left ventricular tissue engineering," Journal of Heart and Lung Transplantation, vol. 21, no. 2, pp. 233243, 2002.

[115] K. A. Robinson, J. Li, M. Mathison et al., "Extracellular matrix scaffold for cardiac repair," Circulation, vol. 112, no. 9, pp. I135-I143, 2005.

[116] K. Matsubayashi, P. W. Fedak, D. A. Mickle, R. D. Weisel, T. Ozawa, and R. K. Li, "Improved left ventricular aneurysm repair with bioengineered vascular smooth muscle grafts," Circulation, vol. 108, Suppl 1, no. 10, pp. II219-II225, 2003.

[117] K. R. Stevens, K. L. Kreutziger, S. K. Dupras et al., "Physiological function and transplantation of scaffold-free and vascularized human cardiac muscle tissue," Proceedings of the National Academy of Sciences of the United States of America, vol. 106, no. 39, pp. 16568-16573, 2009.

[118] J. C. Chachques, J. C. Trainini, N. Lago, M. CortesMorichetti, O. Schussler, and A. Carpentier, "Myocardial assistance by grafting a new bioartificial upgraded myocardium (magnum trial): clinical feasibility study," Annals of Thoracic Surgery, vol. 85, no. 3, pp. 901-908, 2008.

[119] Z. Xiang, R. Liao, M. S. Kelly, and M. Spector, "Collagengag scaffolds grafted onto myocardial infarcts in a rat model: a delivery vehicle for mesenchymal stem cells," Tissue Engineering, vol. 12, no. 9, pp. 2467-2478, 2006.

[120] G. A. Fishbein and M. C. Fishbein, "Arteriosclerosis: rethinking the current classification," Archives of Pathology and Laboratory Medicine, vol. 133, no. 8, pp. 1309-1316, 2009.

[121] R. N. Mitchell and P. Libby, "Vascular remodeling in transplant vasculopathy," Circulation Research, vol. 100, no. 7, pp. 967-978, 2007.

[122] F. J. Schoen and R. J. Levy, "Calcification of tissue heart valve substitutes: progress toward understanding and prevention," Annals of Thoracic Surgery, vol. 79, no. 3, pp. 1072-1080, 2005.

[123] R. A. Hopkins, A. L. Jones, L. Wolfinbarger, M. A. Moore, A. A. Bert, and G. K. Lofland, "Decellularization reduces calcification while improving both durability and 1-year functional results of pulmonary homograft valves in juvenile sheep," Journal of Thoracic and Cardiovascular Surgery, vol. 137, pp. 907-913, 2009.

[124] M. Writing Group, D. Lloyd-Jones, R. Adams et al., "Heart disease and stroke statistics_-2009 update: a report from the american heart association statistics committee and stroke statistics subcommittee," Circulation, vol. 119, no. 3, pp. e21e181, 2009.

[125] P. T. Kovanen, "Mast cells and degradation of pericellular and extracellular matrices: potential contributions to erosion, rupture and intraplaque haemorrhage of atherosclerotic plaques," Biochemical Society Transactions, vol. 35, no. 5, pp. 857-861, 2007. 


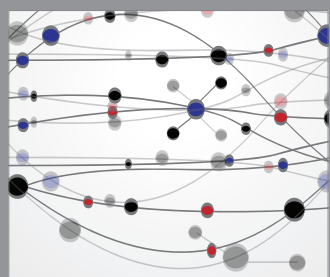

The Scientific World Journal
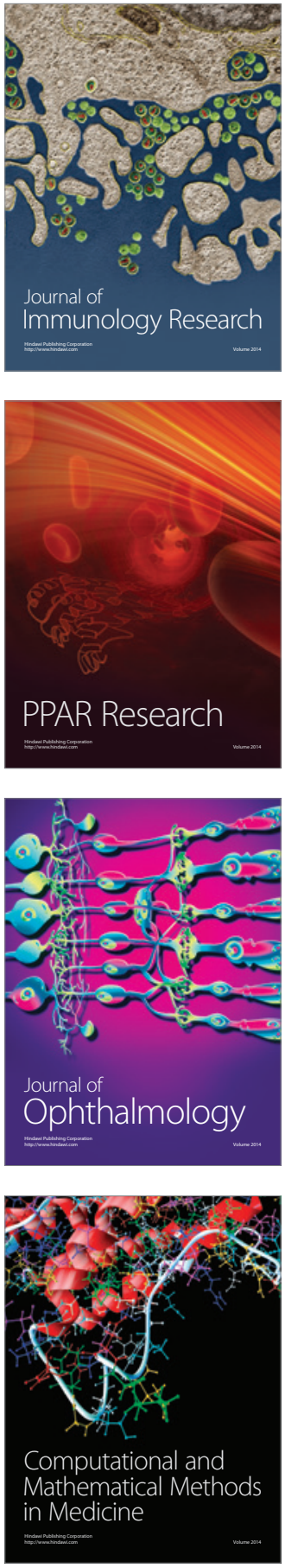

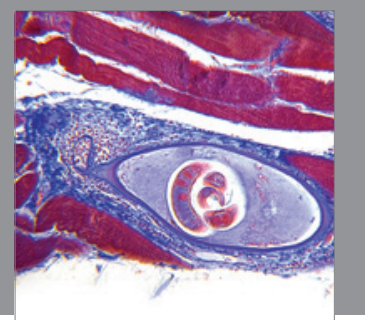

Gastroenterology

Research and Practice
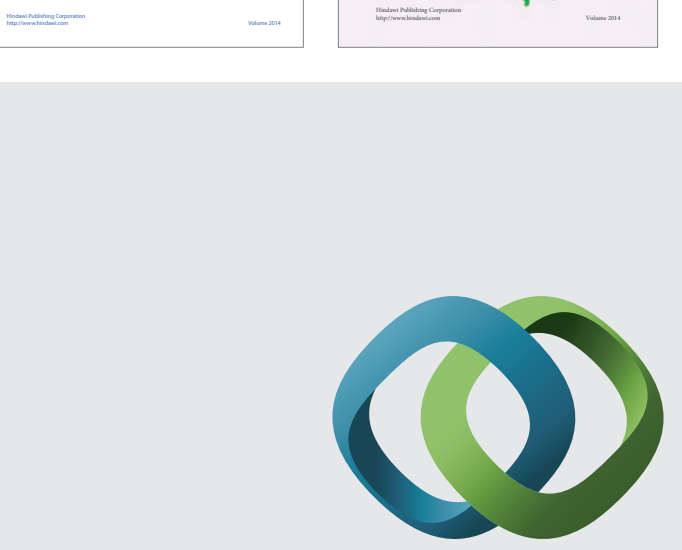

\section{Hindawi}

Submit your manuscripts at

http://www.hindawi.com
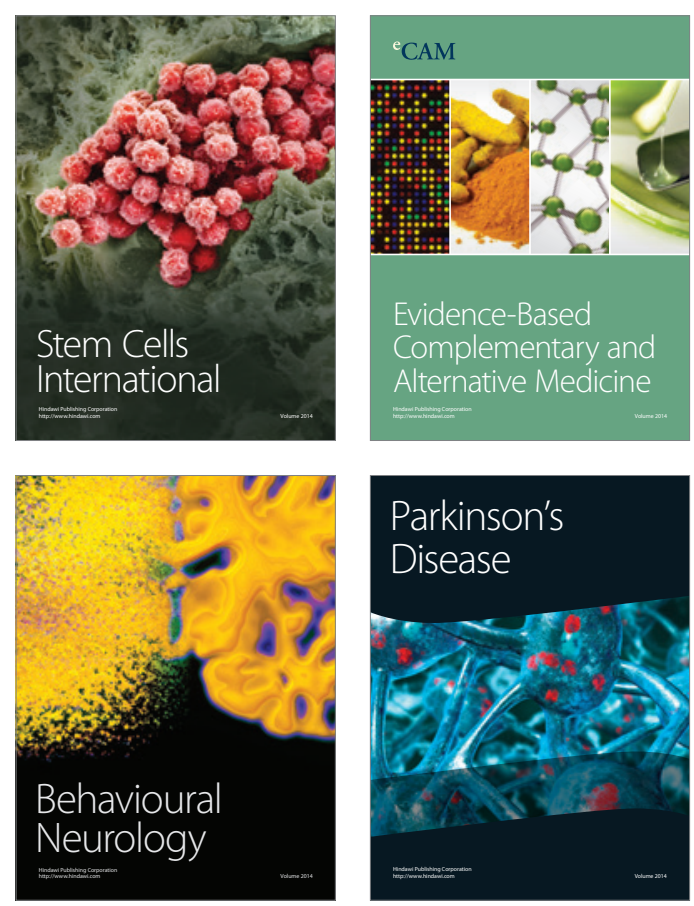

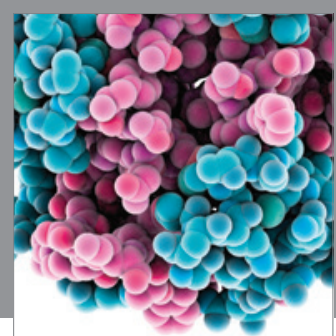

Journal of
Diabetes Research

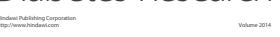

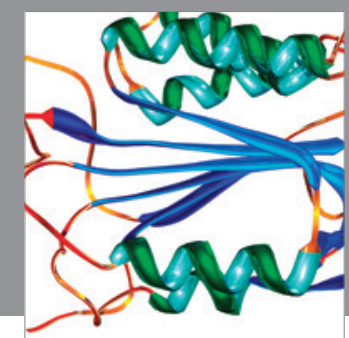

Disease Markers
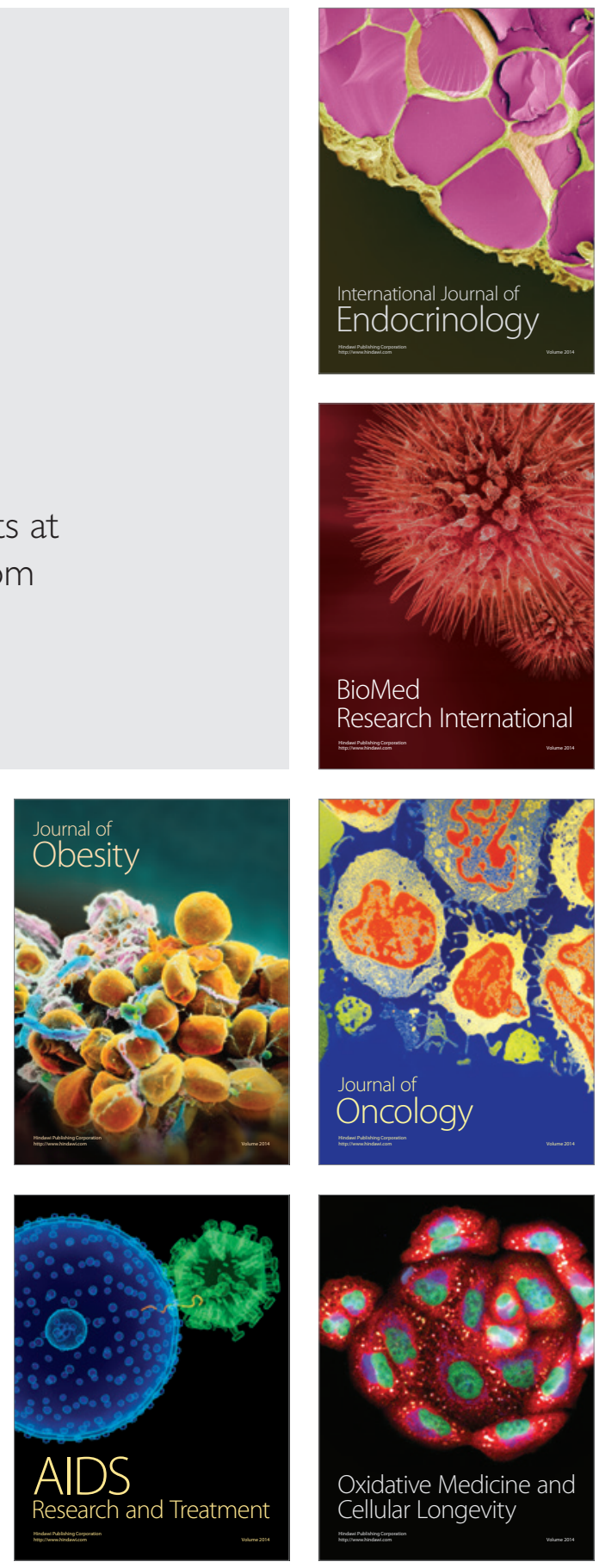